\title{
Communication Blackout and an Alternative Measures for Harsh Space Weather
}

\author{
Anmol Sharma \\ Department of Electronics and Communication Engineering, \\ Indira Gandhi Delhi Technical University for Women \\ Delhi-110006, India
}

\begin{abstract}
This paper has its focus on the Solar Cycle 24, its effects, of which the title, Communication blackout is the major effect. Also it focuses on the hazards caused by a storm and its effects on the space communications system which causes blackouts. This research is based on studies and observations of the previous cycles and their effects. The current cycle which peaks around August 2012, will have disastrous effects. The only solution to this major problem is use of lasers and optical fibre.
\end{abstract}

Keywords : Solar cycles, Satellite, Optical fibre.

\section{INTRODUCTION:}

In telecommunication, communications blackouts are ,the cessation of communication or communications capability, caused by a lack of power to a communications facility or to communications equipment i.e. a total lack of radio communications capability, caused by ionosphere anomalies, e.g., during strong aurorally activity or during re-entry of a spacecraft into the Earth's atmosphere. The Sun follows a regular $11( \pm 1)$ year cycle from a minimum to maximum state of changing magnetic field marked by change in the number of sunspots. Some argue over the cycle to be a $22( \pm 2)$ year long, i.e. minimum to minimum. The Sun performs a cycle from a minimum to maximum solar activity which is observed using the basic and simplest parameter, the number of sunspots visible. During the maxima of this cycle, a high solar activity is observed. High radiation storms from the Sun consisting of super charged protons and other sub atomic particles occur in the space. These storms disturb the magnetic fields of planets and in cases even tear them apart. The Solar cycle was first discovered by Samuel H. Schwabe in 1843 over 17 years of observations. These studies were then compiled by Rudolf Wolf reconstructing the cycle back to 1745 , the time when
Galileo and other contemporaries first observed sunspots. The ongoing cycle which began in December 2008, 6 months late as predicted is expected to peak around August 2012 rather than the earlier prediction of March 2012. Its possible effects are on a rise already, the major being a blackout. The 1859 storm shorted out telegraph wires, causing fires in North America and Europe, sent readings of Earth's magnetic field soaring, and produced northern lights so bright that people read newspapers by their light.

In today's age, there is a huge dependence on satellites for the major part of human communication needs. These satellites are highly prone to the harsh weather occurring in space. This Space weather is generated majorly by the Sun in the form of Solar Wind or a Coronal Mass Ejection (CME) also known as a Geo-Magnetic Storm. These storms cause the satellites to fail causing communications blackout. The model explained is a very basic version of what can be done in such an event. This research should prove useful for the oncoming possible crisis. The ongoing cycle which began in December 2008, 6 months late as predicted is expected to peak around August 2012 rather than the earlier prediction of March 2012. Its possible effects are on a rise already, the major being a blackout. The Carrington event of 1859 shorted out telegraph wires, caused transformer fires in North America and Europe, and the readings of Earth's magnetic field peaked [1].

\section{SOLAR CYCLE:}

The solar cycle, or the solar magnetic activity cycle, is the main source of periodic solar variation driving variations in space weather. The cycle is observed by counting the frequency and placement of sunspots visible on the Sun.

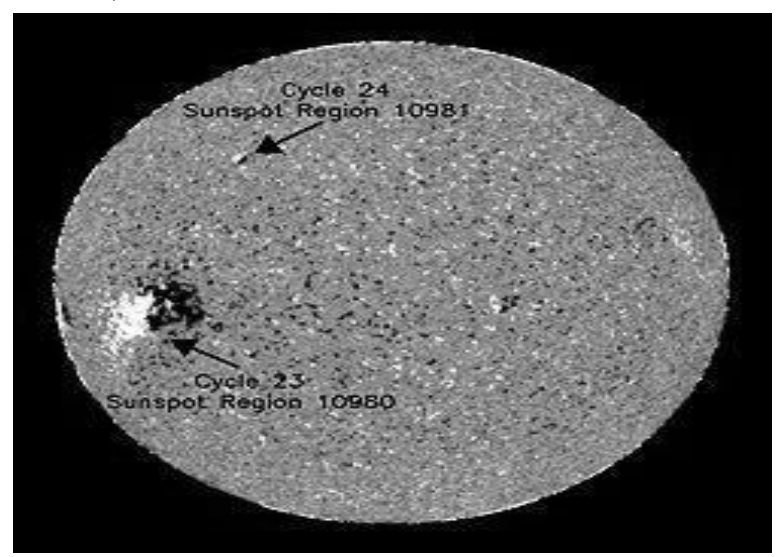

Figure 1: Sunspot on Sun [2] 
Powered by a hydro-magnetic dynamo process driven by the inductive action of internal solar flows, the solar cycle:

Structures the Sun's atmosphere, corona and wind; Modulates the solar irradiance;

Modulates the flux of short-wavelength solar radiation, from ultraviolet to $\mathrm{X}$-ray;

Modulates the occurrence frequency of flares, coronal mass ejections, and other geo-effective solar eruptive phenomena;

Indirectly modulates the flux of high-energy galactic cosmic rays entering the solar system.

"More than 35 years ago, I began drawing the attention of the space physics community to the 1859 flare and its impact on telecommunications," says Louis. Lanzerotti, retired Distinguished Member of Technical Staff at Bell Laboratories and current editor of the journal Space Weather. He became aware of the effects of solar geomagnetic storms on terrestrial communications when a huge solar flare on August 4, 1972, knocked out longdistance telephone communication across llinois. That event, in fact, caused AT\&T to redesign its power system for transatlantic cables. A similar flare on March 13, 1989, provoked geomagnetic storms that disrupted electric power transmission from the Hydro Québec generating station in Canada, blacking out most of the province and plunging 6 million people into darkness for 9 hours; aurora-induced power surges even melted power transformers in New Jersey. In December 2005, X-rays from another solar storm disrupted satellite-to -ground communications and Global Positioning System (GPS) navigation signals for about 10 minutes[3].

\section{SATELLITE INFLUENCE:}

Solar storms are capable of injecting particles and high value currents in the ionosphere, which induce major alterations in the geomagnetic field. Lanzerotti points out that as electronic technologies have become more sophisticated and more embedded into everyday life; they have become more vulnerable to solar activity. There exists a direct proportionality between the sun-spot number, the conductor, the voltage and the Geomagnetic ally Induced Currents. The telecommunications industry relies on the satellites to generate $\$ 168$ billion (The Space Report 2011) in profits each year. Virtually every class of operating satellite has demonstrated a vulnerability to space weather storms. Most are minor, but many involve consequences leading to satellite damage or failure. The orbit of the International Space Station (ISS) is very sensitive to the level of solar storms which cause excess atmospheric drag.

Technology advancements in VLSI has allowed spacecraft components to become smaller, their miniaturized systems have become increasingly vulnerable to the more energetic solar particles. These particles can cause physical damage to microchips and can change software commands in satellite-borne computers. During geomagnetic storms, there is an increase in the number and energy of electrons and ions in the atmosphere. When a satellite travels through this energized environment, the charged particles striking the spacecraft cause different portions of the spacecraft to be differentially charged. Eventually, electrical discharges can arc across spacecraft components, harming and possibly disabling them. Bulk charging or deep charging occurs when energetic particles, primarily electrons, penetrate the outer covering of a satellite and deposit their charge in its internal parts. If sufficient charge accumulates in any one component, it may attempt to neutralize by discharging to other components. This discharge is potentially hazardous to the satellite's electronic systems. In the satellites, the unprotected parts are the damage prone regions by the high energy protons. Deep Dielectric Charging is the reason for failure most of the times. The electrons cause surface charging of satellites similar to static charge being gained by a glass rod. Gold foils are used to cover the highly sensitive parts. One thing that prevents shielding all the parts is the high cost involved. Observations of the last few years show satellite communications systems have experienced signal fluctuations, blackouts and communication failures because of space-weather effects. The frequency range the Defence Department uses widely, which is between $245 \mathrm{MHz}$ to 3 $\mathrm{GHz}$ (VHF to UHF), has found to be very vulnerable to solar activity. Large Solar Energetic Particle (SEP) events can provide significant achievements to the atmospheric secondary registration in the near earth environment, imposing hazards in space operations, major effects being the release of trapped particles from the magnetosphere to aurorally zones causing increased spacecraft charging, interference with satellite communications and surveillance systems., increased satellite drag, loss of magnetic torque attitude control system of satellite, single-event effects in microelectronics, and hazards to space hardware. SEP radiation at lower energies affects the electronics circuits, solar cells and mirrored surfaces [4].

\section{EFFECTS:}

\section{Technological Break-down:}

Our highly technological world is particularly exposed to the electromagnetic effects of space weather. Each solar storm, for example, generates intensive showers of particles and gigantic currents in the ionosphere which induce major alterations in the geomagnetic field. Electric conductors in the changing magnetic field, whether cables, pipes or seawater, run currents called "geomagnetic ally induced currents", or GICs. The bigger the sunspot and the longer the conductor, the higher the voltage and possibly the GIC. While there are natural electric currents running through the earth and sea known as "telluric currents", they vary wildly during coronal mass ejections.

\section{Satellite Communications Network:}

Currently, there are 936 operating satellites in space with a replacement cost of $\$ 200$ billion. The telecommunications industry relies on them to generate $\$ 250$ billion in profits each year. Virtually every class of operating satellite has demonstrated a vulnerability to space weather storms. Most 
are minor, but many involve consequences leading to satellite damage or failure. The orbit of the International Space Station (ISS) is very sensitive to the level of solar storms which cause excess atmospheric drag.

For satellites, the most destructive ingredient of solar storms seems to be in high-energy electrons rather than the other types of particles. These electrons do their damage by producing "deep dielectric charging" in unprotected parts of the satellite. One thing that prevents shielding these parts is the high cost involved.

It's entirely possible and quite likely that during the next few years, satellite-communications systems may experience signal fluctuations, blackouts and communications outages because of space-weather effects. Communications in the frequency range between 245 megahertz and three gigahertz (very high frequency to ultra-high frequency) - a range the Defence Department uses widely - appear to be very vulnerable to solar activity. The same solar-weather-related changes that affect communications also affect the time it takes signals to traverse the ionosphere. The abnormal time delays introduce position errors and decrease the accuracy and reliability of the Global Positioning System, which is used for many range-finding and navigational purposes.

In summary, space-weather -related disruptions to SATCOM systems have wide-ranging effects - from interrupted phone calls to disruption of automated teller machines and problems in global economic transactions. Before deployment, leaders with SATCOM equipment should make a point to minimize the adverse impacts weather could have on their communications and operational readiness [5].

\section{PROPOSED SOLUTION:}

\section{Laser Communication}

The Bhabha Atomic Research Centre, India has developed a low cost semiconductor laser communicator for transmission of computer and telemetry data. It can be easily installed at a short notice provided the line of sight is achieved. The system consists of two sets of transceivers units, which can transmit $10 \mathrm{~K}$ bits/sec of digital information for a range of $1.5 \mathrm{~km}$. Each transmitter emits IR pulses of $200 \mathrm{nsec}$ duration with peak power of $4 \mathrm{~W}$. At the receiver end, the light pulses are focused on a photodiode with the help of suitable optics, which converts them into electrical pulses. These pulses are further processed for interfacing with the computer. The data is exchanged at two ends without laying any cable. Due to the unique property of a very low divergence of a laser beam, the system offers secure communication of data as compared to a radio link.

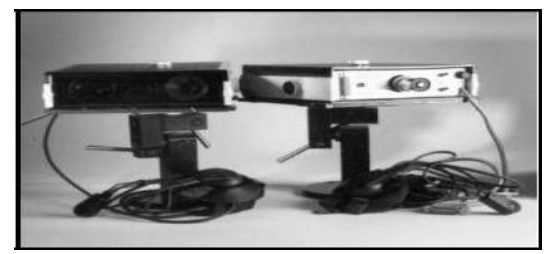

Figure 2: Laser communication developed at BARC, India [6].
Communication technology has experienced a continual development to higher carrier frequencies of several hundred terahertzes since lasers are employed in fibre systems. The main driving force was that the usable bandwidth - and hence transmission capacity - increases proportional to the carrier frequency. Another asset comes into play in free-space point-to-point links. The minimum divergence obtainable with a freely propagating beam of electromagnetic waves scales proportional to the wavelength. The jump from microwaves to light waves therefore means a reduction in beam width by orders of magnitude, even if we use transmitting antennas of much smaller diameter. The reduced beam width does not only imply increased intensity at the receiver site but also reduced cross talk between closely operating links and less chance for eavesdropping.

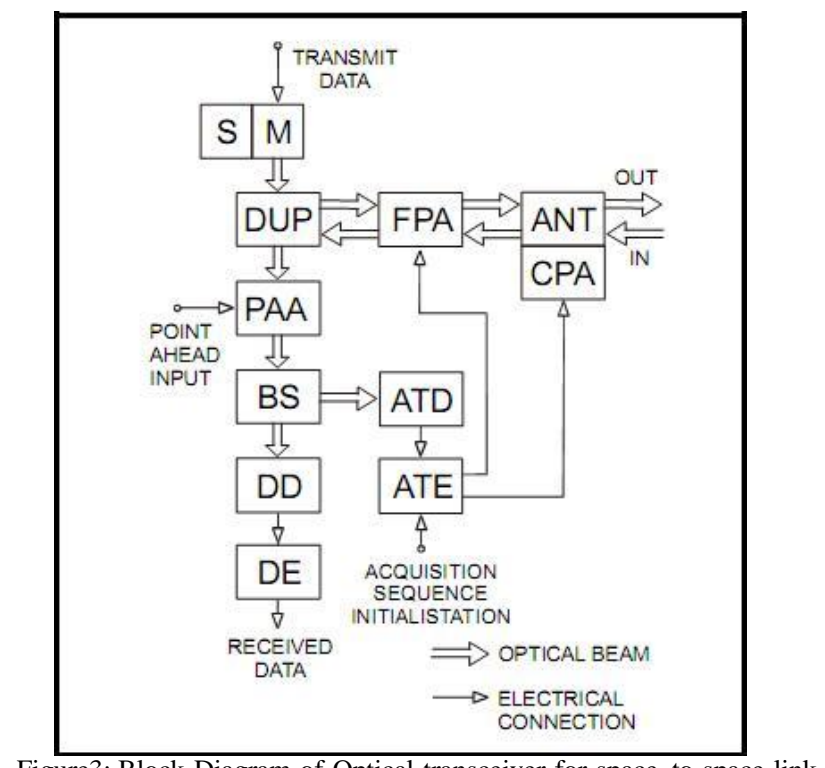

[7].

Free space links follow a certain protocol during communication. The transreceiver section is described in Fig.3. where, S - laser source, M - modulator, DUP optical duplexer, FPA - fine pointing assembly, ANT antenna, CPA-coarse pointing assembly, PAA - point ahead assembly, BS -beam splitter, DD - data detector, DE - data electronics, ATD - acquisition and tracking detector, ATE - acquisition and tracking electronics.

\section{Optical Fibres:}

Optical fibres have proven to be the best form of communication medium for secure transmission of data. In the considered scenario, normal fibres would prove to fail the minimum requirements. Some applications involve exposure to UV or nuclear radiation. These include spectroscopy/ lithography, solar collection, sensors in nuclear reactors, and detectors for accelerators and military vehicles. Fibre performance is typically dependent on glass properties. Because of increased radiation-induced defects in the core, silica can experience dramatic attenuation increases under the radiation, rendering the fibre useless. For UV wavelengths below about $350 \mathrm{~nm}$, a high $-\mathrm{OH}-$ content-core/glass-clad fibre can be used. For UV 
wavelengths below about $250 \mathrm{~nm}$ or under nuclear radiation, the choice of fibre type can be complex, so it's best to consult directly with a fibre manufacturer who specializes in UV transmitting fibres or radiation resistant fibres.

\section{THE PROPOSED MODEL:}

When a solar storm event occurs, in order to shield the satellites from the dielectric charging, it is necessary to power them down. While in this condition, they are to be replaced with an alternate link in order to process the highly critical communication. The basic model shown in Fig.4 includes a terrestrial optical mesh network. This has to be used to stop the radiation from interfering with the ground communications. This network is connected to another domain over the horizon via a mid-space repeater (MSR).

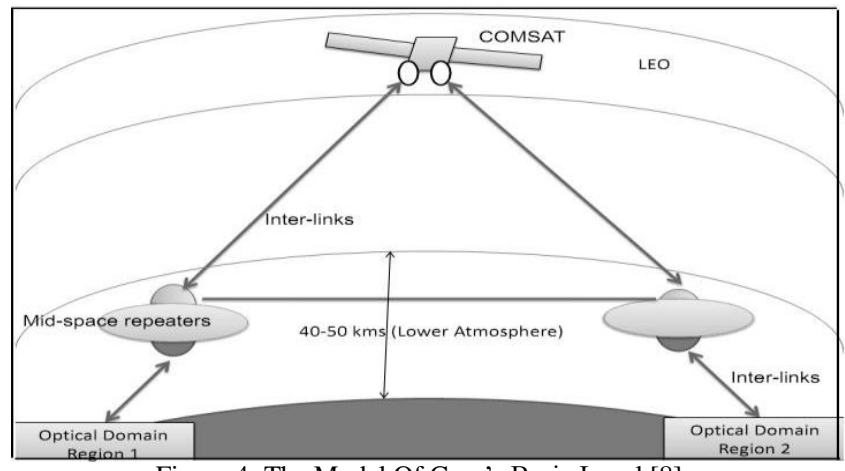

Figure 4: The Model Of Core's Basic Level [8].

The basic model (above) includes a terrestrial optical mesh network. This is to stop the radiations from interfering with the ground communications. This network is connected to another domain over the horizon via a mid-space repeater. These repeaters can be hot air balloons, mini aircrafts, UAVs, etc. These have to be shielded from the external environments by placing them in radiation proof cases. This setup takes up to 30mins from launch to transmission. This is to be used once the CME has passed the Earth's magnetosphere. These repeaters help in overcoming the Earth's curvature effect. The only disadvantage for this is a line of sight communication is necessary. Once the turbulence in the magnetosphere calms down, backup satellites or mini COMSATS kept ready for launch must be fired. These are to be set up at the LEO patch. Thus during the CME event, the communication is restored. The model has been thought over several times to eliminate the possibility of a failure.

\section{DETECTION:}

Measurements of the variations of the earth's magnetic field are handled by several national agencies. These include NOAA"s Space Environment Centre in Boulder, Colorado, Canada's Geological Survey in Ottawa, and the Finnish Meteorological Institute in Helsinki. Once the measurements are made, they are interpreted in terms of the $\mathrm{Ak}, \mathrm{K}$, or $\mathrm{G}$ scales, according to where they are measured on the planet's surface. Agencies such as the National Oceanic and Atmospheric Agency and the Solar and Heliospheric.
Observatory can observe and predict solar activity's impact on the earth. Army leaders responsible for communications should be aware of how to retrieve the results of NOAA ${ }^{\text {ee }} \mathrm{s}$ and $\mathrm{SOHO}^{\text {ee }}$ s observations and take appropriate action if at all possible. Data dumps from the satellites are done every 15 minutes.

Another indicator of the level of solar activity is the flux of radio emission from the Sun at a wavelength of $10.7 \mathrm{~cm}$ (2.8 GHz frequency). This flux has been measured daily since 1947. It is an important indicator of solar activity because it tends to follow the changes in the solar ultraviolet that influence the Earth's upper atmosphere and ionosphere. Many models of the upper atmosphere use the $10.7 \mathrm{~cm}$ flux (F10.7- Frequency at noise level) as input to determine atmospheric densities and satellite dragto follow the sunspot number quite closely and similar prediction techniques can be used.

\section{CONCLUSION:}

Using a communications link that is shielded from radiations emitted during the solar storms will prove to be of great importance during the time of the blackout. As even the power grids will be affected by these storms, all the equipment which consumes high power will be of no use. The system is to be designed in a manner that uses low power supply which can be obtained through stored DC sources and if required can be converted to AC. Backup power would be one of the major requirements which could, in its absence, render the entire model to be useless. To make the system work, the proposed plan of action is: 1 . Prepare the optical terrestrial network and prepare Com Sats ready to launch, 2. Shutdown Satellites as an Earthdirected flare is recorded. 3. Initialize the repeaters and set them in orbit. 4. Launch the Com Sats once the flare has passed the Earth's orbit. This flow of events ensures the communications link to be operational at all stages. The possibility of a failure cannot be ignored, design flaws exist which are to be developed with better resources.

What proposed here is actually a laser link to be used in place of our normal RF link. This will be a large scale application, and have cross country, inter \& intra city links. The optical domain is to be used on the terrestrial level within organizations. Their electronic domains will have to be enclosed in casings so as to protect them from radiation hazards. Eminent researchers like Louis J. Lanzerotti propose to have a new improved fleet of COMSATS ready for launch in order to replace the damaged ones during the event.

\section{REFERENCES:}

[1] Interplanetary medium condition effects in south Atlantic Magnetic Anomaly, Aracy Mendes Da Costa, A case study ,Journal Of Atmospheric And Solar Terrestal Physics Volume 73 ,Issue-11,pp.1478-1491.

[2] Laser Satellite Communications: Current Status and Directions, G. Hyde and B. I. Edelson, A Critical Case Study- Space Policy, 13 April ,2008 , pp.78-112.

[3] Trudy-E.Bell-Dr.Tony.Phillips-“A-Super-Solar-Flare”05.06.2008

[4] Secure Interferometric Communications In Free Space: Enhanced Sensitivity For Propagation In The Metre Range ,F. J. Duarte, Journal of Optics A: Pure and Applied Optics,Volume 10,Issue 2,Janurary 2009. 
[5] Mild Solar Storm Season Predicted But Major Damage Still possible and Magnetic cycle activity, Dr.K.L.Jamaia , Insurance Journal, Volume 4,No.2,12 May 2009,pp.21 -34.

[6] Understanding Space Weather Shield Society, Brain Dunbar, A technical Report conducted by NASA, Pages Editor Steve Fox ,Volume 98, No.72,13 January 2010.

[7] Stormy Weather: Solar Activity could Wreak Havoc on Satellites, Debbie Linton, Magazine-Army Communicator, Winter Edition ,Volume 2,Issue 1, 3 February 2010.pp.125- 146.

[8] Its Only Rocket Science: An Introduction In Plain English Astronomers' Universe: Springer eBooks collection, Springer ISBN 978-0-387-75378-2, Volume 45, June 2010, pp. 159-162.

[9] The Rise and Rise of Optical Fibers,Gambling, W. A , IEEE Journal on Selected Topics in Quantum Electronics, Vol. 6, No. 6, Nov./Dec. 2010,pp.1084-1093.

[10] Future Giant on Laser Communication, Senoir Sciencetist, Dr J.P. Singh, News-BARC Newsletter, Newsletter Of Founder's Day (Mumbai),Bhabha Atomic Research centre, 29 October 2010.pp-7-10

[11] An Overview - Optical Fibre and its Performance, Nagel S. R, Mac Chesney J. B Walker

[12] K. L, IEEE Journal of Quantum Electronics, Vol. QE-18, No. 4 , April 2011,pp.54-112.

[13] Research and Development of Free-Space Laser Communications and Quantum Key Distribution Technologies at NICT,M. Toyoshima, T. Sasaki, H. Takenaka, Y. Shoji, Y. Takayama, Y. Koyama, H. Kunimori, M. Akioka, M. Fujiwara and M. Sasaki, Proceedings of IEEE ICSOS, 20 May 2011.

[14] Space Laser Communication System,Technologies ,Satellites and Application, Review of laser engineering, Dr.Daniel ,Analytical Report conducted by NASA, Volume.28,No.12, November 2011, pp.804-808.20.

[15] NOAA Space Weather Scale for Radio Blackouts , J.P Thomas, NOAA -Space Weather Predictions, Volume 17, No.7, 14Januray 2012,pp.15-45.

[16] James Goulding, Research -II Sun(Flux Of radio Emission at a wavelength of $10.7 \mathrm{~cm}$ - www.jamesgoulding.com), James Goulding , Magazine-MIT Area Scope ,Volume 59,Issue 15,September 2012,pp.659-678.

[17] Fibres For Harsh Environments, H.Carol, MagazineEnvironmental challenges, Volume 4,Issue 2,14 November 2012,pp.245-252.

[18] GPS World News and Application on Global Positioning System, Joseph Marie (IIT, Delhi),Digi-Magazine, Volume 32,Issue 1December 2013,pp.25-28.

[19] Space Weather, Dr .Rudolf Kreutzer, IEEE, Journal of Space Communication Signal, Volume 17, No. 6, 8 December 2013.pp.90-145.

[20] Space Exploration Technologies , Emine Richard, IEEE Journal Of Aerospace and Navigation, Volume 13,Issue 7,December 2014.

[21] Significant Achievements in Space Communications and Navigation,NASA Spacex, Volume $\quad 67$, No.18,March 2015,pp 30-32. 